\title{
El polimorfismo de la IL-1 y su eventual asociación con la enfermedad periodontal crónica. Una revisión de la literatura (II)
}

\author{
VALDERRAMA G* \\ VIJANDE $\mathbf{F} * *$ \\ ESCRIBANO JM $* * *$ \\ GARRIDO-PERTIERRA $\mathbf{A} * * * *$ \\ BASCONES $\bar{A} * * * * *$
}

Valderrama G, Vijande F, Escribano JM, Garrido-Pertierra A, Bascones A. La IL-1 y su eventual asociación con la enfermedad periodontal crónica. Una revisión de la literatura (I). Av Periodon Implantol. 2005; 17, 3: 157-163.

\section{RESUMEN}

El propósito de esta revisión es describir el papel que desempeña el polimorfismo de los genes de la IL- 1 en la aparición y evolución de la enfermedad periodontal. A lo largo de ella, se observa una falta de homogeneidad en los resultados obtenidos por los diferentes autores. Incluso los estudios más recientes tienden a desestimar la relación que pudiera existir entre la presencia de polimorfismos en los genes de la IL-1 y la enfermedad periodontal. De igual manera, la presencia de estos polimorfismos parece no influir en el fracaso implantario ni en las técnicas de regeneración tisular guiada.

\section{PALABRAS CLAVE}

Interleuquina, IL-1, enfermedad periodontal crónica, polimorfismo.

Aceptado para publicación: Abril 2005

\section{INTRODUCCIÓN}

Dentro de la respuesta inflamatoria del huésped, tres son los productos bioquímicos que están fuertemente asociados a la severidad y progresión de la enfermedad periodontal. Las Interleuquinas (IL-1), las prostaglandinas (PGE2) y las metaloproteinasas de la matriz (MMPs). De estas sustancias, nos hemos centrado en la IL-1 y en particular en su forma beta. Numerosas estirpes celulares son capaces de secretar la IL-1, pero su producción viene determinada genéticamente por los genes IL-1A, IL-1B e IL-1RN, localizadas en la posición $2 q 13$ y muy próximos entre sí. Éstos, genes son polimórficos y por tanto susceptibles de sufrir cualquier tipo de variación genética. El polimorfismo genético es un tipo de mutación monogénica, en el que se produce la sustitución de un par de bases nitrogenádas por otro par distinto. Ello, puede originar la sobreproducción de la proteína codificada lesionando los tejidos de inserción del diente.

Diferentes estudios han demostrado que la IL-1 $\beta$ es 15 veces más potente que la IL-1 $\alpha$ y 500 veces más que la TNF $\alpha$ y la linfotoxina en la mediación de la

* $\quad$ Odontólogo. Master en Operatoria y Estética Dental por UMS.

** Odontólogo. Master en Periodoncia e Implantes por UCM.

*** Médico. Jefe de Servicio del Instituto de Cardiología de la Comundiad de Madrid.

**** Doctor en Farmacia y en Veterinaria. Catedrático de Bioquímica y Biología Molecular del Departamento de Bioquímica y Biología Molecular IV de la Facultad de Veterinaria de la UCM.

***** Doctor Médico-Estomatólogo. Catedrático de Medicina Bucal y Periodoncia del Departamento de Medicina y Cirugía Bucofacial de la Facultad de Odontología de la UCM. 


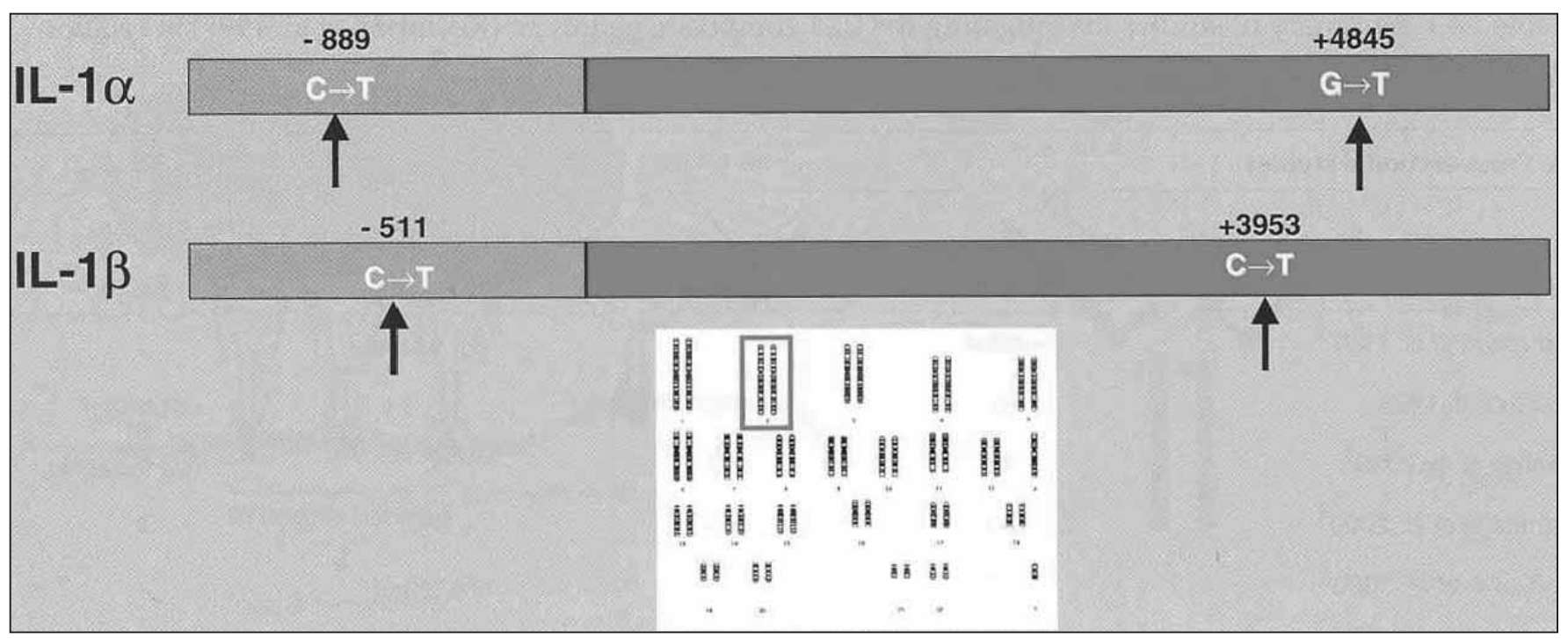

Fig. 1: Los genes para la $I L-A$ y la IL-B, localizados en el cromosoma 2. Se indican con flechas negras donde se encontrarían los posibles polimorfismos de ambos genes.

reabsorción ósea in vitro (1). Además la IL-1 $\beta$ y el TNF $\alpha$ se han aislado en fluído gingival crevicular (FGC) de lugares con periodontitis, en mayor cantidad que la IL- $1 \alpha(2,3)$. Estas cantidades de IL- $1 \beta$ son de 10 a 50 veces mayores con respecto a la IL-1 $\alpha$ (4). Estas son dos de las principales razones por las que nos decantamos por esta forma de la proteína.

Según Kornman (5), se considera a un individuo "genotipo positivo" para los genes de la IL-1, cuando poseen al menos un polimorfismo (alelo 2) en uno de sus dos alelos para el gen de la IL-1A y al menos un polimorfismo (alelo 2) en uno de sus dos alelos para el gen de la IL-1B. El término "alelo 2" se emplea para definir la segunda forma más frecuente de encontrarnos una secuencia de nucleótidos de un determinado alelo.

Relación del genotipo positivo con la respuesta inflamatoria periodontal. Numerosos artículos han demostrado una clara asociación entre el estado periodontal del paciente y una mayor concentración de IL-1 en el fluido crevicular, en especial de su forma beta.

Stashenco y col. (6), tomaron muestras de IL-1 $\beta$ en tres zonas diferentes; con actividad de la enfermedad, con inactividad de la enfermedad y lugares sanos, todas estas muestras se tomaron de 12 pacientes con enfermedad periodontal del adulto. Observaron que en las zonas con actividad de la enfermedad, la concentración de IL- $1 \beta$ aislada era significativamente mayor al de las zonas sanas. Otros artículos con un diseño similar han llegado a los mismos resultados $(7,8,9)$. Gonzáles y col. (10) en un modelo de estudio de gingivitis experimental, tomaron muestras a 12 pacientes sanos de IL-1 $\beta$ en FGC en los días $0,7,14$ y 18. Demostraron claramente un incremento en la concentración de IL-1 $\beta$ en las zonas con gingivitis experimental. Idénticos resultados han obtenido otros investigadores en estudios similares $(11,12)$. Numerosos autores han llegado a la conclusión de que la concentración de IL- $1 \beta$ en zonas sanguinolentas con enfermedad periodontal, es aproximadamente 10 veces mayor que en zonas sanas $(13,14,15)$. Referente a la importancia del tabaco en las concentraciones de IL-1 $\beta$ y de IL-lra en GCF, Boström y col. (16), realizaron un estudio donde tomaron muestras a 22 pacientes periodontales fumadores y a 18 pacientes periodontales no fumadores. Tras analizar las muestras de IL- $1 \beta$ y de IL-lra, observaron que no existían diferencias significativas en cuanto a las concentraciones que presentaban los dos grupos de estudio. La IL-l y el TNF- $\alpha$ son potentes estimuladores de la reabsorción ósea. Por lo tanto, una sobreproducción de cualquiera de estas dos citoquinas provocada por algún tipo de polimorfismo genéti$\mathrm{co}$, se cree que pueda ser uno de los mecanismos responsables de la destrucción del tejido periodontal. Así mismo, un estudio experimental realizado en monos, observó que la inhibición simultánea de la IL-1 y del TNF, prevenía en su mayor parte la reabsorción ósea de la enfermedad periodontal (17).

Algunos individuos, que producen mayor cantidad de IL-1 que otros, no sólo lo hacen esporádicamente en una medición concreta, sino que sus niveles de IL- 1 se mantienen constantemente incrementados. Además está particularidad, tiende a heredarse dentro de una misma familia. Hoy en día, sabemos que las variaciones en ciertos genes de la IL-1, son responsables de provocar una sobreproducción de esta proteína al estar el individuo expuesto a una carga bacteriana. En 
algunos estudios in vitro, se procedió a aislar células sanguíneas de la serie blanca, las cuales fueron incubadas en el laboratorio junto a productos de bacterias gram-negativo, observándose significativamente una mayor producción de IL-1 $\beta$ en aquellas células procedentes de individuos con genotipo positivo $(18,19,20)$. Pociot y col. (20) realizaron un estudio in vitro acerca de la producción de IL-1 $\beta$ por parte de monocitos de pacientes de los cuales no se conocía su estado periodontal. Informaron que los individuos heterocigóticos compuestos (al menos un alelo 2) para el gen de la ILlB +3953 , producían entre un $30-40 \%$ más de IL- $1 \beta$ frente a LPS de E.coli que los individuos genotipo negativo para el mismo polimorfismo. Mientras que los individuos homocigóticos para el gen de la IL-1B +3953 , producían un $50 \%$ más de IL- $1 \beta$ que los individuos genotipo negativo. En un estudio reciente, Engebreston y col. hallaron que los niveles de IL-1 $\beta$ en FGC eran significativamente mayores en pacientes genotipo positivo, que en pacientes genotipo negativo (21). De hecho, las diferencias más significativas entre los pacientes genotipo positivo y genotipo negativo, se encontraron en bolsas periodontales $<4 \mathrm{~mm}$. En las zonas donde se presentaban las formas leve-moderada de la enfermedad, los individuos genotipo positivo, presentaban valores de IL-1 $\beta$ en FGC 2.5 veces mayores que individuos genotipo negativo con una $(\mathrm{p}=$ 0.03).

En otro estudio, Shirodaria y col. (22) observaron que el presentar un polimorfismo genético denominado alelo 2 en el locus del gen IL-1A, en su posición -889, está asociado a un incremento en los niveles de la proteína IL- $1 \alpha$. Este incremento de proteína en pacientes con periodontitis severa es aproximadamente cuatro veces mayor de lo normal. Estas diferencias se evidencian, con mayor claridad, en individuos no fumadores. Kornman y col. (5) realizaron un estudio con sujetos no fumadores del norte europea con polimorfismo compuesto de la IL- $1 \alpha$ en la posición -889 y el de la IL-1 $\beta$ en la posición +3953 (genotipo positivo), y observaron que el polimorfismo era significativamente más frecuente en sujetos con periodontitis avanzada $(67 \%$ OR=18.9), que en sujetos con periodontitis leve o sin enfermedad periodontal (22\%). Este estudio sugiere que el llevar el alelo 2, tanto en forma homocigótica como heterocigótica en ambos locus, predispone en mayor medida a estos individuos a desarrollar la enfermedad periodontal en su estado severo. Otro estudio realizado por McDevitt y col. (23) con no fumadores o ex fumadores con una historia de $<5$ paquetes/año, investigaron la presencia de polimorfismos en otro conjunto de genes, IL- $1 \alpha(+4845)$ e IL-1 $\beta$ (+3954). Concluyeron que ser portador de ambos polimorfismos, supone un riesgo mayor $(\mathrm{OR}=3.8-5.3)$ de padecer enfermedad periodontal severa, en comparación con los sujetos sin variaciones genéticas. En otros dos estudios $(24,25)$ donde sólo se observó el polimorfismo del gen IL-1B en la posición +3953 , se encontró que existía una clara relación entre dicho polimorfismo genético y el padecer la enfermedad periodontal del adulto. A pesar de incluir pacientes fumadores en el estudio, fueron capaces de encontrar diferencias significativas. Por el contrario estudios más recientes como el realizado por Mark y col. (18) muestran que la producción de IL$1 \beta$ por parte de monocitos aislados, que fueron enfrentados a patógenos periodontales, no presentaron diferencias estadísticamente significativas en cuanto al genotipo positivo o negativo para los genes de la IL-1. Santtila y col. (26) también observaron una disminución en la producción de IL-1 $\beta$ por parte de células mononucleares periféricas en aquellos individuos que presentaban polimorfismo en el gen IL-1B en la posición +3953 . No se ha encontrado una explicación plausible para estas discrepancias, aunque podría ser que la producción de la IL- $1 \beta$ esté regulada por otro locus genético además de por el locus +3953 del gen de la IL-1B.

Relación del genotipo positivo con la prevalencia de la enfermedad periodontal. Rogers y col. (27) no encontraron diferencias significativas en cuanto al polimorfismo en el gen IL-1A en la posición +4845 o a ser genotipo positivo entre un grupo con enfermedad peridontal crónica, otro con periodontitis rápidamente progresiva y un tercer grupo de pacientes sanos. Por otro lado, también observaron que la posesión de polimorfismos en los genes de la IL-1 no aumentaba la susceptibilidad en la pérdida de implantes. Otros autores como Papapanou y col. (28) fueron incapaces de encontrar diferencias estadísticamente significativas respecto a poseer un genotipo positivo o negativo entre un grupo de individuos con enfermedad periodontal y otro grupo de pacientes sanos. Similares resultados encontraron Pociot y col. (20), quienes determinaron que la gravedad de la enfermedad periodontal era la misma tanto para individuos con genotipo positivo como con genotipo negativo. Por el contrario, Galbraith y col. (24), incluyendo fumadores en sus grupos de estudio, observaron una relación estadísticamente significativa entre el hecho de presentar polimorfismos en el gen de la IL-1B en la posición +3953 y el padecer la enfermedad periodontal en un estadio avanzado. Estos autores concluyen que los individuos con este tipo de polimorfismos son tres veces más susceptibles de padecer la enfermedad que aquellos que no presentan dicho polimorfismo. Gore y col. (25) en un estudio donde también se incluían pacientes fumadores, también encontraron una mayor frecuencia de polimorfismos en el gen IL-1B en la posición +3953 en pacientes con enfermedad periodontal severa. Aunque estas diferencias no fueron estadísticamente significativas, estos resultados difieren de los encontrados por Kornman y col. (5). Estos autores encon- 
traron diferencias estadísticamente significativas entre pacientes con enfermedad periodontal grave no fumadores y el hecho de presentar polimorfismos genéticos. Pero esta asociación, sólamente aparecía cuando los polimorfismos se encontraban tanto en el gen IL-1A como en el gen IL-1B (genotipo positivo). Por otro lado, Shirodaria y col. (22) contemplaron una asocicación estadísticamente significativa entre la presencia de polimorfismos en el gen IL-1A en la posición +4845 y un mayor riesgo de padecer enfermedad periodontal grave en pacientes no fumadores. Aunque esta asociación sólamente la observaron en el gen IL-1A, no descartaron que aquellos individuos que además presentasen otro polimorfismo en el gen IL-1B, podrían manifestar una mayor predisposición de padecer la enfermedad. Así mismo, McDevit y col. (23) a pesar de encontrar diferencias entre los grupos con enfermedad periodontal y control respecto a ser genotipo positivo o negativo, estas diferencias no fueron estadísticamente significativas. Aún así, consideran el hecho de poseer polimorfismos en los genes de la IL-1 un manifiesto factor de riesgo de padecer la enfermedad periodontal crónica. Por el contrario, cuando sólo tuvieron en cuenta a los pacientes no fumadores, las diferencias observadas si fueron estadísticamente significativas entre los dos grupos. Por tal razón, ha de tenerse en cuenta la importancia del tabaco en la etiología de la enfermedad. Cullinan y col. (29) concluyen que el hecho de ser genotipo positivo para los genes de la IL-1 contribuye a una mayor susceptibilidad de adquirir la enfermedad periodontal, pero no es un factor esencial en el desarrollo de la misma. Thomson y col. (30) describen una asociación entre un determinado compuesto genotípico, (homocigótico para el alelo- 1 en el gen IL- 1 A +4845 y homocigótico para el alelo-2 en el gen IL-1B +3953) y una mayor prevalencia de la enfermedad periodontal en individuos con 26 años de edad. Aunque sugieren que estos resultados podrían estar sujetos a algún tipo de error debido al diseño del estudio. Jepsen y

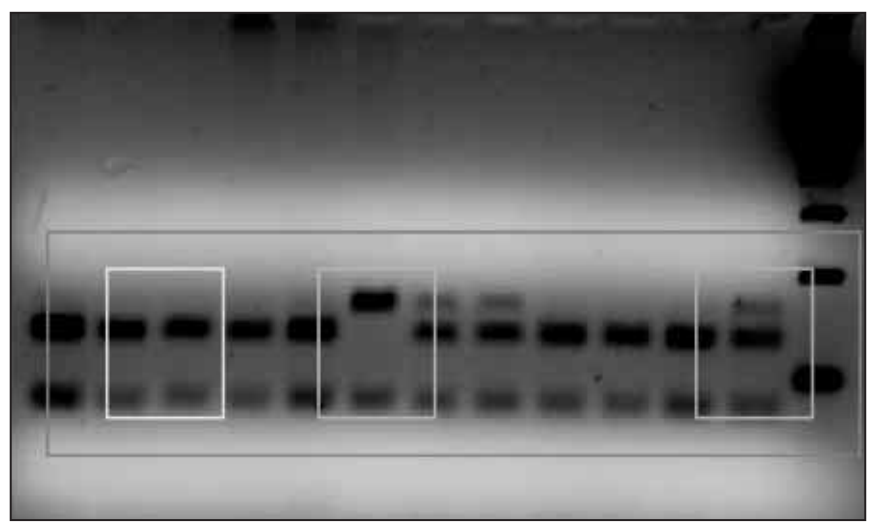

Fig. 2: Digestión enzimática del producto de PCR del gen IL- 1 A en un gel de agarosa al 3\%. col. (31) fueron incapaces de hallar la influencia del factor genético de la IL-l referente a la aparición de la gingivitis. En un estudio bastante completo realizado en el 2002, Meisel y col. (32) no encontraron correlación entre ser genotipo positivo para la IL-1, presentar polimorfismo en el gen IL-1B +3953 o presentar polimorfismo en el gen IL-1A +4845 y una mayor pérdida ósea o cualquier otro parámetro clínico periodontal estudiado. Posteriormente, estos mismos autores (33) observaron que sólamente los pacientes fumadores con genotipo positivo para la IL-1 poseían un mayor riesgo de padecer la enfermedad periodontal que los controles. Concluyen que debe existir una asociación sinérgica entre el factor genético y el tabaco. Siguiendo con esta pauta, en el 2004 Meisel y col. (34) han reincidido que los pacientes con genotipo positivo para la IL-1, que eran fumadores, poseían un mayor riesgo de padecer la enfermedad periodontal. Concluyen que el tabaco constituye un factor de riesgo más importante que el factor genético, puesto que en las personas sin ese hábito, el factor genético es incapaz de expresarse y por tanto, desaparece su implicación en la aparición y progresión de la enfermedad.

Teniendo en cuenta los trabajos observados en esta revisión bibliográfica, podemos concluir que los estudios más recientes, tienden a no considerar la relación entre la presencia de polimorfismos en los genes de la IL-1 y la prevalencia de la enfermedad periodontal, afirmando que los polimorfismos en los genes de la IL-1 no son unos marcadores genéticos fiables para determinar la susceptibilidad de la enfermedad periodontal.

Relación del genotipo positivo con el tratamiento periodontal y la osteointegración de los implantes. McGuire y Nunn (35), realizaron un estudio en una población de pacientes que habían sido sometidos a un manteniemiento periodontal por un periodo de tiempo de entre 5 y 14 años. Evaluaron las causas de

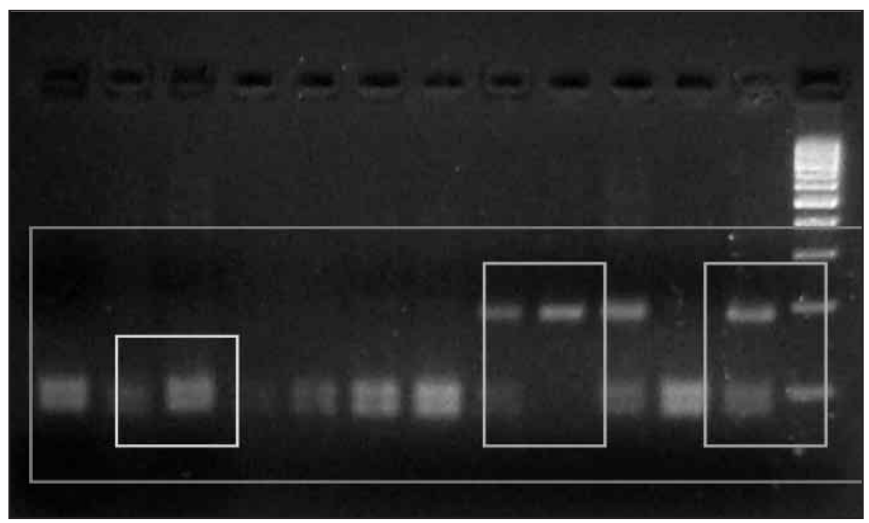

Fig. 3: Digestión enzimática del producto de PCR del gen $I L-1 B$ en un gel de agarosa al 3\%. 
la pérdida dentaria, tras el tratamiento periodontal completo y durante las fases de mantenimiento. Estos investigadores sólo encontraron dos factores significativos capaces de predecir la pérdida dentaria. Por un lado el ser genotipo positivo para los genes de la IL-l y por otro, el ser un paciente gran fumador (más de 40 paquetes/año). El poseer un genotipo positivo para los genes de la IL-l, incrementó el riesgo de pérdida dentaria en 2.7 , mientras que el ser un paciente gran fumador (más de 40 paquetes/año) lo incrementó en 2.9. Además, en aquellos pacientes que siendo grandes fumadores presentaban un genotipo positivo para los genes de la IL-1, el riesgo de pérdida dentaria se incrementaba hasta en un 7.7. El resto de parámetros clínicos tradicionalmente usados para determinar el pronóstico de un diente, sólo fue válido para los pacientes genotipo negativo en los genes de la IL-1 y que además fuesen no fumadores. Lang y col. (36) realizaron un estudio en una población de pacientes en mantenimiento periodontal. A los 323 pacientes del estudio, se les practicó un examen periodontal que incluía mediciones de profundidad de sondaje y de zonas sangrantes tras el sondaje. Además se les tomó una muestra sanguínea para determinar su genotipo para los genes de la IL-1A y de la IL-1B. Dentro del grupo de pacientes no fumadores, se encontraron diferencias significativas $(p=$ 0.03 ) entre los individuos genotipo positivo para el gen de la IL-l y un incremento en las zonas sangrantes tras el sondaje. De hecho, los pacientes genotipo negativo para el gen de la IL-l presentaban un 50\% menos de zonas sangrantes tras el sondaje. Estos autores concluyen que el aumento de zonas sangrantes tras el sondaje en sujetos genotipo positivo, prueba que estos individuos presentan una respuesta inflamatoria genéticamente predeterminada. La cual influirá en la respuesta clínica de los tejidos periodontales. En otro estudio, DeSanctis y col. (37) se evaluaron los resultados obtenidos tras el empleo de técnicas quirúrgicas de regeneración tisular guiada en zonas con pérdida de inserción periodontal. En ninguno de los 40 pacientes tratados con cirugía, se detectaron diferencias significativas en cuanto a los resultados clínicos, después del primer año del tratamiento. A los cuatro años de la cirugía de regeneración, los pacientes que presentaron un genotipo negativo para los genes de la IL-1, manifestaron un $73 \%$ de zonas estables tras el tratamiento (se pierde menos de $1 \mathrm{~mm}$ de inserción clínica). En cambio, los pacientes que presentaron un genotipo positivo para los genes de la IL-1, manifestaron tan sólo un $21.4 \%$ de zonas estables tras el tratamiento de regeneración. Actualmente los implantes dentales se han convertido en una parte integral de la periodoncia y la odontología restauradora. Sin embargo, a pesar de las recientes publicaciones acerca de la predictibibilidad de los tratamientos con implantes, existen muy pocos estudios relacionando la influencia de la gené- tica con el éxito de los implantes. En uno de estos estudios, Thomas y col. (38) investigaron la relación existente entre diferentes factores tales como: el genotipo de la IL-1, el hábito de fumar y la edad con respecto a la supervivencia de los implantes valorando su osteointegración. Se compararon 27 pacientes que habían perdido 33 implantes, o al menos perdido un $50 \%$ de hueso alrededor del mismo, con 38 pacientes sin pérdida ósea o de implantes. Se demostró que el tabaco incrementaba el riesgo de pérdida de implantes en al menos 2.5. Por el contrario, no se detectaron diferencias significativas en los otros dos factores que se estudiaron, ni la edad, ni el tener genotipo positivo para los genes de la IL-l incrementaban el riesgo de pérdida de implantes. Rogers y col. (39) en un estudio más reciente, tampoco encontraron diferencias significativas entre pacientes de raza caucasiana con genotipo positivo para la IL-1A (899) y para la IL-1B (+3953) ni un mayor riesgo de pérdida de implantes. Por el contrario, Feloutzis y col. (40) realizaron un estudio cuyos objetivos se centraban en: primero, investigar la relación entre el polimorfismo específico de los genes de la IL-1 y la pérdida ósea periimplantaria en implantes dentales ITI ${ }^{\circledR}$, y segundo, en explorar la asociación entre ésta variación alélica de los genes de la IL-1 y la inflamación periimplantaria de la mucosa. Su población de estudio constaba tanto de individuos fumadores como no fumadores. Demostraron en aquellos pacientes con un gran hábito de fumar y que además presentaban polimorfismo positivo en los genes de la IL-1 un mayor riesgo de sufrir pérdida ósea periimplantaria después de la rehabilitación protésica y durante las fases de mantenimiento periodontal.

\section{SUIMIMARY}

The aim of this bibliographic review is describing the role of the polymorphism in IL-1 genes in the appearance and evolution of the periodontal disease. Through the review, it's observed a lack of homogeneity in the results obtained by different authors. Despite of this, more recent articles tend to have a low opinion of the relationship that could exist between the presence of polymorphism in IL- 1 genes and periodontal disease. In the same way, the presence of theses polymorphisms seems not to influence in the implant failure nor in the guided tissue regeneration techniques.

\section{KEY WORDS}

Interleukin, IL-1, chronic periodontal disease, polymorphism. 


\section{BIBLIOGRAFÍA}

1. Stashenko P, Dewhirst EE, Peros WJ, Kent RL, Ago JM. Synergistic interaccions between interleukin 1 , tumor necrosis factor, and lymphotoxin in bone resorption. J Immunology 1987; 138: 1464-8.

2. Jandinski. JJ, Rynar JE, Steinle M, Leung CE, Deasey.M.J, Stashenko P. Localization of Interleukin $l \alpha$ and tumor necrosis factor $\alpha$ in human gingiva. J Dent Res 1989; 68. (Special Issue) abstr 526.

3. Jandinski JJ, Stashenko P, Feder LS, Leung CC, Rynar JE, Deasy MJ. Localization of interleukin-1 beta in human periodontal tissue. J Peririodontol 1991;62: 36-43.

4. Tatakis DN. Interleukin-1 and bone metabolism: a review. Periodontol 1993; 64: 416-4.

5. Kornman KS, Crane A, Wang HY, di Giovani FS, Newman MG, Pirk FW, Wilson TG, Higginbottom FL, Duff GW. The interleukin-1 genotype as a severity factor in adult periodontal disease. J Clin Periodontol 1997; 24: 72-7.

6. Stashenco P, Fujiyoshi P, Obernesser MS, Prostak L, Haffajee $A D$, Socransky SS. Levels of interleukin $1 \beta$ in tissue from sites of active periodontal disease. J Clin Periodontol 1991; 18: 548-54.

7. Masada MP, Persson R, Kenney JS, Lee SW, Page RC, Allison AC. Measurement of interleukin-1 alpha and 1 beta in gingival crevicular fluid: implications for the pathogenesis of periodontal disease. J Periodont Res 1990; 25: 156-63.

8. Preiss DS, Meyle J. Interleukin-1 beta concentration of gingival crevicular fluid. J Periodontol 1994; 65: 423-8.

9. Tsai CC, Ho YP, Chen CC. Levels of interleukin-8 in gingival crevicular fluids in adult periodontitis. J Periodontol 1995; 66: 852-9.

10. Gonzáles JR, Hermann JM, Boedeker RH, Franez PI, Biesalski H, Meyle J. Concentration of interleukin- $1 \beta$ and neutrophil alastase activity in gingival crevicular fluid during experimental gingivitis. J Clin Periodontol 2001; 28: 544-9.

11. Heasman PA, Collins JG, Offenbacher S. Changes in crevicular fluid leveles of interleukin-1 beta, leukotriene B4, prostaglandin E2, thromboxane B2 and tumor necrosis factor alpha in experimental gingivitis in humans. J Periodont Res 1993; 28: 241-7.

12. Kinane DF, Winstanley FP, Adonogianaki E, Moughal NA. Bioassay of interleukin 1 (IL-1) in human gingivitis crevicular fluid during experimental gingivitis. Archives of Oral Biology 1992; 37: 153-6.

13. Rawlinson A, Dalati MHN, Rahman S, Fairclough AL. Interleukin-1 and IL-1 recep-tor antagonist in gingival crevicular fluid. J Clin Periodontol 2000; 27: 738-43.

14. Lee HJ, Kang IK, Chung CP, Choi SM. The subgingival microflora and gingival crevicular fluid cytokines in refractary periodontitis. J Clin Periodontol 1995; 22: 885-90.
15. Ishihara Y, Nishihara T, Koroyanagi T, Shirozu N, Yamagishi E, Ohguchi M, Koide M, Ueda N, Amano K, Noguchi T. Gingival crevicular interleukin-1 and interleukin-1 receptor antagonist levels in periodontally healthy and diseased sites. J Perio-dont Res 1997; 32: 524-9.

16. Boström L, Linder LE, Bergström J. Smoking and GCF levels of IL-1 $\beta$ and IL-lra in periodontal disease. J Clin Periodontol 2000; 27: 250-5.

17. Assuma R, Oates T, Cochran D, Amar S, Graves DT. IL-1 and TNF antagonists inhibit the inflammatory response and bone loss in experimental periodontitis. J Immunol 1998; 160: 403-9.

18. Mark LL, Haffajee AD, Socransky SS, Kent Jr RL, Guerrero D, Kornman K, Newman M, Stashenko P. Effect of the interleukin-1 genotype on monocyte IL-lbeta expression in subjects with adult periodontitis. J Periodont Res 2000; 35: 172-7.

19. di Giovine FS, Cork MJ, Crane A. Novel genetic association of an IL- $1 \beta$ gene variation a +3953 with IL- $1 \beta$ protein production and psoriasis. Cytokine 1995; 7: 606.

20. Pociot F, Molving J, Wogensen L. A Taql polymorphism in the human interleukin-1 beta (IL-lbeta) gene correlates with IL-1 beta secretion in vitro. Eur J Clin Invest 1992; 22 : 396-402.

21. Engebrestson SP, Lamster IB, Herrera-Abreu M, et al. The influence of interleukin gene polymorphism on expression of interleukin- $1 \beta$ and tumor necrosis factor- $\alpha$ in periodontal tissue and gingival crevicular fluid. J Periodontol 1999; 70: 567-73.

22. Shirodaria S, Smith J, Mckay IJ, Kennett CN, Hughes FJ. Polymorphisms in the IL-l $\alpha$ gene are correlated with levels of interleukin-l alpha protein in gingival crevicular fluid of teeth with severe periodontal disease. J Dent Res 2000; 79: 1864-9.

23. McDevitt MJ, Wang HY, Knobelman C, Newman MG, di Giovine FS, Timms J, Duff GW, Kornman KS. Interleukin-1 genetic association with periodontitis in clinical practice. J Periodontol 2000; 71: 156-63.

24. Galbraith GMP, Hagan C, Steed RB. Cytokine production by oral and peripheral blood neutrophils in adult periodontitis. J Periodontol 1997; 68: 832-8.

25. Gore E, Sanders JJ, Pandey JP, Palesch Y, Galbraith GMP. Interleukin- $1 \beta+3953$ allele 2 : association with disease status in adult periodontitis. J Clin Periodontol 1998; 25: 781-5.

26. Snattila S, Savinainen K, Murme M. Presence of the IL-1RA allelo 2 (ILI-1RN*2) is associated with enhance IL- $1 \beta$ production in vitro. Scand J Immunol 1998; 47: 195-8.

27. Rogers MA, Figliomeni L, Baluchova K, Tan AES, Davies G, Henry PJ, Price P. Do interleukin-1 polymorphisms predict the development of periodontitis or the success of dental implants?. J Periodont Res 2002; 37: 37-41.

28. Papapanou PN, Neiderud A-M, Sandros J, Dahlén G. Interleukin-1 gene polymorphism and peridontal status. J Clin Peruidontol 2001; 28: 389-96. 
29. Cullinan MP, Westerman B, Hamlet SM, Palmer JE. Faddy MJ, Lang NP, Seymour GJ. A longitudinal study of interleukin-l gene polymorphisms and periodontal disease in a general adult population. J Clin Periodontol 2001;28: 1137-44.

30. Thomson WM, Edwards SJ, Dobson-Le DP, Tompkins GR, Poulton R, Knight DA, Braithwaite AW. IL-l genotype and adult periodontitis among young New Zealanders. J Dent Res 2001; 80 (8): 1700-3.

31. Jepsen S, Eberhard J, Fricke D, Hedderich J, Siebert R, Acil T. Interleukin-l gene polymorphisms and experimental gingivitis. J Clin Periodontol 2003; 30: 102-6.

32. Meisel P, Siegemund A, Dombrowa S, Sawaf H, Fanghaenel J, Kocher T. Smoking and polymorphisms of the interleukin-l gene cluster (IL- $1 \alpha$, IL- $1 \beta$ and IL-RN) in patients with periodontal disease. J Periodontol 2002; 73: 27-32.

33. Meisel P, Siegemund A, Grimm R, Herrmann FH, John U, Schwahn C, Kocher T. The interleukin-1 polymorphism, smoking, and the risk of periodontal disease in the population based ship study. J Dent Res 2003; 83 (3): 189-93.

34. Meisel P, Schwahn C, Gesch D, Bernhardt O, John U, Kocher T. Dose-effect relation of smoking and the interleukin-1 gene polymorphism in periodontal disease. J Periodontol 2004; 75: 236-42.

35. McGuire MK, Nunn ME. Prognosis versus actual outcome, IV. The effectiveness of clinical parameters and IL-1 genotype in accuarately pre-dicting prognoses and tooth survival. J Periodontol 1999; 70: 49-56.
36. Lang NP, Tonetti MS, Suter J, Sorrell J, Duff GW, Kornman KS. Effect of the interleukin-1 gene polymorphisms on gingival inflammation assessed by bleeding on probing in periodontal maintence population. J Periodont Res 2000; 35: 102-7.

37. DeSanctis M, Zuchelli G. Intrleukin-1 gene polymorphisms and longterm stability following guided tissue regeneration. J Periodontol 2000; 71:606-13.

38. Thomas G, Wilson Jr, Nunn M. The relationship between the interleukin-1 periodontal genotype and the implant loss. Initial data. J Periodontol 1999; 70: 724-9.

39. Rogers MA, Figliomeni L, Baluchova K, Tan AES, Davies G, Henry PJ, Price P. Do interleukin-l polymorphisms predict the development of periodontitis or the success of dental implants?. J Periodont Res 2002; 37: 37-41.

40. Feloutzis A, Lang NP, Tonetti MS, Bürgin W, Brägger U, Duff GW, Kornman KS. IL-1 gene polymorphism and smoking as risk factor for periimplant bone loss in a well maintained population. Clin Oral Impl Res 2003; 14: 10-7.

\section{CORRESPONDENCIA}

Guillermo Valderrama Mateos

c/ Porvenir $n^{\circ} 4$ bajo $A$

Colmenarejo. 28270 Madrid

telf. 918589061 / 649434868 